\title{
Dental practitioners with a special interest in periodontics: the West Sussex experience
}

IN BRIEF

- Shares experiences of a new concept.

- Promotes the role of treatment of periodontal disease in the primary sector. - Explains the benefits of clinical networks.

- Shows the benefits of a behavioural approach.

\author{
P. D. Cheshire, ${ }_{1}^{1}$ P. Saner, ${ }^{2}$ R. Lesley, ${ }_{1}{ }^{3}$. Beckerson, ${ }_{1}^{4}$ M. Butler ${ }^{5}$ \\ and B. Zanjani ${ }^{6}$
}

VERIFIABLE CPD PAPER

\begin{abstract}
The experience of a pilot service involving practitioners with a special interest in periodontics is described. The service functioned as a clinical network between the primary and secondary sector and featured consultant outreach. Between June 2006 and May 2007 it experienced 441 referrals. It improved patient access to periodontal care and was successful in targeting specific disease categories and in meeting key performance indicators. The service was non-surgical and emphasised patient self-efficacy. It produced highly effective clinical outcomes. It was well accepted by both patients and referring practitioners. It did not replace the need for a consultant-led service in the eyes of the referring practitioners. The BPE was used to identify suitable patients; audit indicated that there was a tendency for practitioners to underscore the level of periodontal disease.
\end{abstract}

\section{INTRODUCTION}

The NHS Plan $(2000)^{1}$ set out a commitment to create a more patient-centred health service with better access to improved services and reduced waiting times. In 2004, the Department of Health announced as part of their programme to modernise dentistry ${ }^{2}$ their intention to increase patient choice, provide patients with greater access to care and more fairly remunerate general dental practitioners. Subsequent to this announcement was the imposition of waiting list targets for secondary care providers. The targets for first appointments were reduced year on year and were then extended to treatment provision in 2008.

These reforms were occurring at a time of increasing referrals for restorative dentistry to the Royal West Sussex Trust, as evidenced

'Consultant in Restorative Dentistry, Maxillofacial Unit, St Richard's Hospital, Spitalfield Lane, Chichester, West Sussex, P019 6SE, ${ }^{2}$ Senior Dental Officer, Central Clinic, Stoke Abbot Road, Worthing, BN11 1HE, ${ }^{3}$ General Dental Practitioners, The Croft Dental Practice, 30 Park Drive, Rustington, BN16 3DY, ${ }^{4}$ General Dental Practitioners, Cuckfield Dental Practice, 3 High Street, Cuckfield, RH17 5EN, ${ }^{5}$ General Dental Practitioners, The Bosham Clinic, Main Road, Fishbourne, P018 8AN, ${ }^{6}$ General Dental Practitioners, Henfield Dental Practice, 1 Brick House, High Street, Henfield, BN5 9DB

${ }^{*}$ Correspondence to: Mr David Cheshire

Email: david.cheshire@wsht.nhs.uk

\section{Refereed Paper}

Accepted 24 August 2010

DOI: 10.1038/sj.bdj.2011.48

${ }^{\circledR}$ British Dental Journal 2011; 210: 127-136 by the Trust's referral data. Resource issues in the secondary sector meant that nationwide most trusts produced referral and acceptance criteria in an attempt to match capacity with demand and therefore stay within waiting list targets.

Once treatment provision was made the responsibility of the Primary Care Trusts, there was a drive to move care from what was generally perceived as the more expensive secondary sector into the primary sector.

In restorative dentistry this created problems. Analysis of the Specialist Register in June 2003 revealed that there were 2847 practitioners on the specialist list for restorative dentistry, endodontics, periodontics and fixed and removable prosthodontics. 1404 of the specialists were over 50 years of age, with 2710 of the total number over 40 years of age. The practitioners on the Specialist Register were either part of the secondary sector or were predominantly practising in the private sector.

In May 2004 the Department of Health, in conjunction with the Faculty of General Dental Practice (FGDP), advanced the concept of general dental practitioners with a special interest in a particular aspect of dental care (DwSI) ${ }^{3}$ and subsequently produced a guideline document for implementing a service. ${ }^{4}$
They defined dental practitioners with a special interest as:

'A dentist working in a primary care setting who provides services which are in addition to their usual and important generalist role. The DwSI provides a service which is complementary to the secondary services but does not replace that provided by a dentist who has undergone the training required for entry to a specialist list. The DwSI is an independent practitioner who works within the limits of their competency in providing a special interest service, and who refers on where necessary. The DwSI may deliver a clinical service beyond that normally provided by a primary dental care practitioner or may deliver a particular type of treatment. Individual DwSIs will be able to demonstrate their competencies in their special interest areas. Special interests may be demonstrated by dentists through the completion of formal training programmes and/or experience based evidence.'

Competency framework documents for practitioners with a special interest were drawn up with the help of the specialist societies in the areas of periodontics, endodontics, minor oral surgery and orthodontics.

A study published in 2002 entitled 'Why undertake outreach into general dental practice?' ${ }^{5}$ concluded that there 
were clearly talented General Dental Practitioners (GDPs) working in general practice but that the NHS remuneration could impact upon these talents; that there was evidence that the outreach provided effective teaching, with application of the knowledge gained leading to changes in clinical practice; and that communication between the primary and secondary sectors improved as a result of the outreach.

The outreach experience revealed a number of factors that helped to explain the high referral rate of periodontal patients to the secondary sector. It appeared to the consultant that within most of the practices visited, periodontology had become the 'province' of the hygienist. The hygienist was often working in isolation, with little effective communication between the hygienist and dentist. There was an emphasis on scaling and polishing the dentition, in line with the NHS fee structure. As a consequence little emphasis was placed on behaviour modification to improve patient-performed oral hygiene. In practices where oral hygiene was emphasised, conflicting information was often given to the patients by the different clinicians leading to confusion among patients and little improvement in their oral hygiene.

In general patients were paying lip service to their own role in plaque control and were heavily reliant on the hygienist, believing that a regular visit to a hygienist was crucial to maintaining gingival and periodontal health. It was the experience of the authors of this paper that, despite visiting the hygienist, many patients with periodontal disease had little perception or understanding of their own role in disease management when they were initially referred to the periodontal service. Within all of this, it was evident that there was an increasing 'blame culture' developing in patients diagnosed with chronic periodontitis.

There are many reasons for the above observations; not least among these were the clinical terms and conditions under which periodontal care was delivered in general practice. At that time periodontal care was remunerated at a reasonable level only if a scale and polish had been carried out. In general the situation has not been improved by the new dental contract.

\section{DEVELOPMENT OF A} PERIODONTAL DWSI SERVICE

In 2005 because of the high proportion of periodontal referrals to the local consultant, Mid Sussex Primary Care Trust (PCT) approached one of the authors to discuss the PCT's intention of developing a periodontal DwSI Service. There was reluctance among the five PCTs in West Sussex to increase the resources for the hospitalbased restorative dentistry service as funding streams were being directed toward the primary sector. Practitioners with a special interest in periodontics were seen as a way of boosting the low profile of periodontology within the primary sector.

Among the consultant body and specialist societies there were concerns raised about the development of practitioners with a special interest. These included the following:

- There was a drive within PCTs to reduce treatment costs leading to a 'dumbing down' of service provision

- Secondary sector services were subject to audit, clinical governance and monitoring and at that time, this was not a prominent feature of primary care

- There was a belief among some GDPs that without further training, they are able to deliver comparable or better services than the ones provided in the secondary sector

- Any dentist trained outside the United Kingdom who subsequently provided DwSI services would not reflect the 'philosophy' of the British-trained practitioners

- The potential to remove resources from the secondary sector, leading to undermining of small units and indeed larger teaching hospitals

- PCTs had little concept of the interdependence of funding between the primary sector, the secondary sector and training institutions.

Discussions at the West Sussex Local Dental Committee (LDC) meetings raised the following concerns among practitioners:

- The establishment of DwSIs would confuse patients and lead to unfair competition between practitioners

- Most practitioners felt that the distinction between specialists, DwSIs and general dental practitioners was not well understood by the National Health Service, let alone by the patients

- There was concern over the quality of care that the GDPs' patients would receive and who would be responsible for the consequences of inappropriate care

- There was concern that patients would be encouraged to leave the referring practitioner in favour of the DwSI.

As a means of managing funding and more closely monitoring treatment provision ahead of the new dental contract, Mid Sussex PCT was also planning to introduce a referral management centre (RMC) to handle all dental referrals.

Following discussions between the various groups, a decision was taken to pilot a Practitioner with a Special Interest in Periodontics service funded by Mid Sussex PCT, but with the aim of providing care for all patients in West Sussex, irrespective of their PCT. The local consultant in restorative dentistry was asked to take responsibility for the service philosophy and structure.

\section{Structure of the periodontal DwSI service}

The following aims for the service were identified:

- The provision of an effective but defined periodontal service that at its inception would not include periodontal surgery. Periodontal surgery would continue to be provided in the secondary sector when required

- To be patient-centred with emphasis on behaviour change and patientperformed oral hygiene and self regulation

- Instrumentation would be delayed where possible until there were signs of adherence with plaque control and signs of resolution of the marginal gingival tissues

- To identify patients requiring periodontal therapy in a primary sector setting and those requiring specialist care or treatment provision in the secondary sector

- The creation of a clinical network which would interface between the primary and secondary sectors, rather than the two sectors working 
independently of one another

- The creation of a service framework that facilitated and enhanced periodontal treatment outcomes

- The creation of a service framework that allowed for data capture, audit and clinical governance.

The objectives for the service were to enhance the local provision of periodontal treatment under the NHS by the formation of a local clinical network uniting the primary and secondary sectors. The network would utilise the skills of general dental practitioners with a special interest in periodontics and consultant outreach into the DwSI practices would be an active component of the network. The service would be based in GDP practices but would be partly administered by the PCT via a referral management centre.

The initial scope of the service was to provide periodontal treatment for patients with moderate to advanced chronic periodontitis. Aggressive disease would continue to be treated in the secondary sector. Once disease control had been achieved, the patients would be maintained by a partnership between themselves and their own GDPs.

\section{The DwSI selection process}

Following a local recruitment advertisement, the PCT received 40 expressions of interest. Nineteen practitioners returned the application forms of which nine were invited for interview.

The selection committee comprised the following individuals:

- A representative of the PCT

- The Dental Vice Dean of Kent Surrey Sussex Deanery

- A member of the British Society of Periodontology council

- The local consultant in restorative dentistry.

Five practitioners were selected, taking into consideration the following:

- The National Competency Framework document produced by the Faculty of General Dental Practice

- Key skills in dental practice

- Evidence of continued professional development in periodontology

- The venue of the practice.
Before taking up the position, the GDPs had to agree to attend a weeklong training programme. The Kent Surrey Sussex (KSS) Deanery provided the funding for this training. The deanery accredited the training for 60 hours of continuing professional development (CPD) and also provided recognition of the training hours for other modular courses based at the University of Kent.

The DwSIs were given a contractual framework to provide periodontal care for two half-day sessions per month. The PCT also agreed to fund the DwSIs' attendance at the annual British Society of Periodontology conference, as part of their continuing professional development. The DwSIs were given an annual contract and were expected to undergo a revalidation based on an audit of their performance.

\section{The training needs for the DwSI}

Within the philosophy of the service it was felt that the DwSI would need to develop the following:

- An understanding of periodontal diseases, their presentation and their prevalence within the population

- Communication skills and particularly those related to behaviour modification

- Treatment planning skills in general and periodontal treatment planning skills in particular

- A systematic approach to diagnosis and treatment of periodontal diseases, utilising designated protocols and treatment guidelines, so that outcomes could be properly evaluated across all the providers of the service

- An ability to evaluate the patient response to therapy

- An understanding of the need to record and maintain appropriate data.

The lecture programme was developed to reflect the training aims and included the following topics:

- Diagnosis of periodontal diseases

- The role of risk factors

- Treatment planning of the periodontal patient

- Perio-endo lesions

- Periodontal reassessment

- The evidence base behind the nonsurgical treatment philosophy

- Psychology of chronic illness and how to induce behaviour change
- Effective communication

- How to access evidence-based knowledge.

The programme content was put together based on the current evidence base, the experience gained from the Outreach into General Dental Practice on how to deliver effective periodontal care in this setting. Philip Ower and Graham Smart, specialist periodontal practitioners, refined the content of the periodontal lectures bearing in mind the aims and objectives for the pilot service.

Smoking cessation was a key part of the periodontal training programme and training. Smoking Cessation Advisor training was also given to an identified person within each of the DwSI practices. The newly trained advisors were linked to the Mid Sussex Smoking Cessation Service and the data from the periodontal service was to add to their audit data. Unfortunately, with the reorganisation of the various PCTs into West Sussex PCT, this aspect of the pilot service was discontinued after approximately five months.

In order that the DwSI data could be pooled, a calibration exercise in periodontal probing was undertaken during the course. Analysis of the data from this exercise revealed that pooling the DwSI data would be acceptable. The service data was captured on Software of Excellence. Peter Saner (one of the authors and a DwSI) customised the software to capture predetermined essential data. The use of the software allowed for audit of individuals, as well as pooled data. Part of the training programme involved instruction on the use of the database.

Before the course the DwSIs were sent key papers on biofilms, plaque and the aetiology and pathology of periodontal diseases. The DwSIs' knowledge of the papers was tested by means of a multiple short answer questionnaire (MSA), on the first day. A daily MSA was used to assess the assimilated knowledge of the lectures from the day before. To facilitate this assessment, each lecturer was required to submit at least 10 questions which reflected key points from their lecture(s).

It was a requirement that the DwSI had to pass each MSA at a mark of $75 \%$ or higher in order to pass the training programme and gain DwSI status. It was also 
a requirement that the DwSI attended each day of the course and that they completed their Key Skills (FGDP) assessment by September 2006.

\section{Referral pathway and criteria}

In order to identify suitable patients, a referral form combining the basic periodontal examination and periodontal risk factors was developed.

\section{Acceptance criteria}

- Basic periodontal examination (BPE) category 4

- Patients younger than 35 years of age with a BPE category 3

- Risk factors or drug interactions affecting periodontal disease or its management, for example, diabetes.

In the letter sent to referring GDPs it was explained that their patients would only be accepted on a basis of periodontal treatment need and not for financial reasons. It stated that the referring practitioner was responsible for the overall management of the patient and for periodontal maintenance once the patient has been discharged from the DwSI service.

The referral form was designed so that non-clinical administration staff in the PCT's referral management centre (RMC) could process it. The consultant provided some initial training of the staff and he met with them on a weekly basis in order to manage any difficulties. The need for these meetings decreased as the knowledge and experience of the staff increased.

The referral pathway was therefore from the GDP to the RMC whereupon, if the details indicated that referral to a DwSI was appropriate; the patient was allocated to the DwSI that was nearest to the patient's home address. If this DwSI did not have capacity, the patient would be asked to contact the next nearest DwSI with capacity. If a patient's referral details indicated that the disease was aggressive or severe, the patient was passed directly to the secondary sector services. The breakdown of the service figures is shown in Table 1.

\section{Service criteria and ethos}

Key to the ethos of the DwSI service was a uniform message and care pathway. Patient information leaflets on the nature

\begin{tabular}{|c|c|c|c|c|c|c|}
\hline Month & $\begin{array}{l}\text { Referrals } \\
\text { sent to } \\
\text { DwSI }\end{array}$ & $\begin{array}{l}\text { Referrals } \\
\text { returned } \\
\text { GDP }\end{array}$ & $\begin{array}{l}\text { Referrals } \\
\text { direct to } \\
\text { consultant }\end{array}$ & $\begin{array}{l}\text { Declined } \\
\text { referrals as } \\
\text { out of area }\end{array}$ & $\begin{array}{l}\text { Referral } \\
\text { resubmitted } \\
\text { by GDP }\end{array}$ & $\begin{array}{l}\text { Referral not } \\
\text { allocated by } \\
\text { PCT }\end{array}$ \\
\hline May-07 & 8 & 5 & 0 & 1 & 0 & 51 \\
\hline Apr-07 & 43 & 2 & 1 & 2 & 0 & 4 \\
\hline Mar-07 & 24 & 3 & 0 & 0 & 1 & 0 \\
\hline Feb-07 & 39 & 11 & 4 & 0 & 0 & 0 \\
\hline Jan-07 & 18 & 1 & 0 & 0 & 0 & 0 \\
\hline Dec-06 & 27 & 6 & 1 & 2 & 1 & 0 \\
\hline Nov-06 & 37 & 4 & 0 & 0 & 1 & 0 \\
\hline Oct-06 & 19 & 6 & 2 & 1 & 2 & 0 \\
\hline Sep-06 & 20 & 1 & 1 & 1 & 1 & 0 \\
\hline Aug-06 & 19 & 2 & 1 & 2 & 2 & 0 \\
\hline Jul-06 & 13 & 5 & 1 & 2 & 3 & 0 \\
\hline Jun-06 & 21 & 9 & 1 & 7 & 2 & 0 \\
\hline Total & 288 & 55 & 12 & 18 & 13 & 55 \\
\hline
\end{tabular}

and treatment of periodontal disease and the role of a practitioner with a special interest were drawn up by the consultant. Photographs of the DwSI were placed on the front page of the DwSI leaflet.

Following allocation to a DwSI, the DwSI leaflet with the appropriate photograph was sent to the patient by the RMC, along with the contact details of the DwSI. The RMC also contacted the DwSI. On receipt of the information from the RMC, the DwSI had ten days to organise a consultation appointment. The compliance to this time frame was also audited.

Following the consultation, the DwSI gave the leaflet on periodontal disease to the patient. The leaflet reinforced the need for self-regulation and the significance of properly performed daily oral hygiene. It also allowed the patient a period of reflection on the information given to them by the DwSI.

For the service to function effectively with the resource available and the likely demand, patient throughput was critical. The clinical care protocol is shown in Figure 1.

Patients who are having difficulty adhering to an oral hygiene regime or who are receiving maintenance therapy can easily prevent new patient access to a periodontal service. It was therefore important that non-adherent patients were firstly identified and then returned back to their GDPs. Patients unable to achieve plaque scores of $15 \%$ or less within four visits were considered in this category. The standard was made explicit to the patient at the consultation and it was also explicit that failure to attend an appointment without explanation would prevent any further appointments within the service.

Periodontal maintenance was not part of this service and patients were returned back to their practitioners for this aspect of care. The lack of maintenance within this service therefore increased the emphasis on the need to achieve behaviour change as part of the long term management of their disease.

\section{Periodontal probing}

There was much discussion about the value and validity of periodontal probing at an initial consultation. As there was a need to validate and audit the referring GDP's BPE score, a level of probing was felt necessary. From the point of view of producing audit and monitoring data, a six-point pocket chart and a BPE score, along with a plaque score and a bleeding score, were performed at the first visit. This acted as the baseline data. The calibration exercise performed during the training exercise indicated that it was acceptable to combine the data for the DwSIs. 


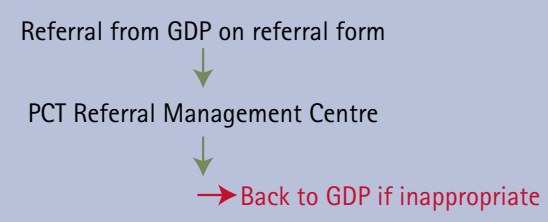

Allocation of patient to DwSI

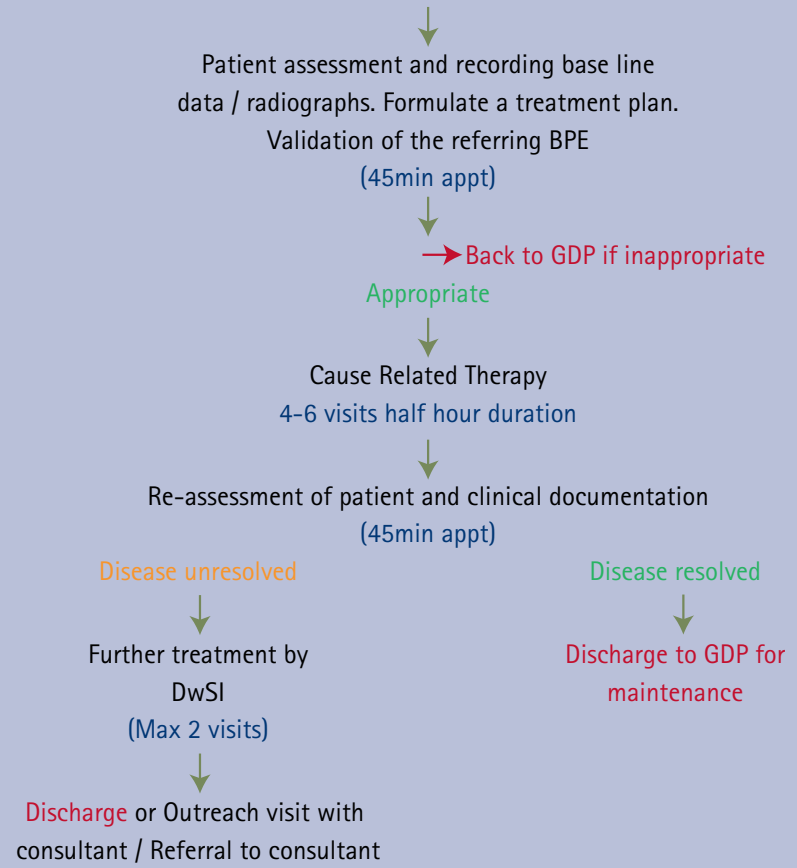

Fig. 1 The clinical protocol/pathway

Patients whose disease levels at the consultation were found to be below the acceptance criteria for the service were given oral hygiene instruction and sent back to the referring practitioner. Any patient whose disease level was considered by the DwSI to be of a more aggressive nature was forwarded to the secondary sector after the initial cause-related therapy. The validity of the referring GDP's BPE was audited and is presented in the results and discussion section (Fig. 2).

\section{Correspondence}

The DwSI had a duty of care to keep the referring GDPs informed regarding their patients' progress. DwSI were required to correspond with the referring practitioner at the following stages:

- After the consultation

- On discharge

If any emergency care became necessary during the course of the treatment, the service specification limited the DwSI to periodontal care with temporisation of teeth only when necessary.

\section{AUDITING THE SERVICE}

Audit of the service was facilitated by adaptation of the Software of Excellence program and was carried out on four levels:

- Does the service work for the PCT?

- Does it work for the patients?

- Does it work for the referring GDPs?

- Does it work for the DwSIs?

The last category was facilitated by regular feedback meetings as a group (including the consultant) and by the consultant outreach. The audit included monitoring of key performance indicators of the DwSI as a whole and as individuals, as well as the PCT.

\section{Method for data capture}

\section{Referrals}

The referral management centre logged the number of patients referred to the centre

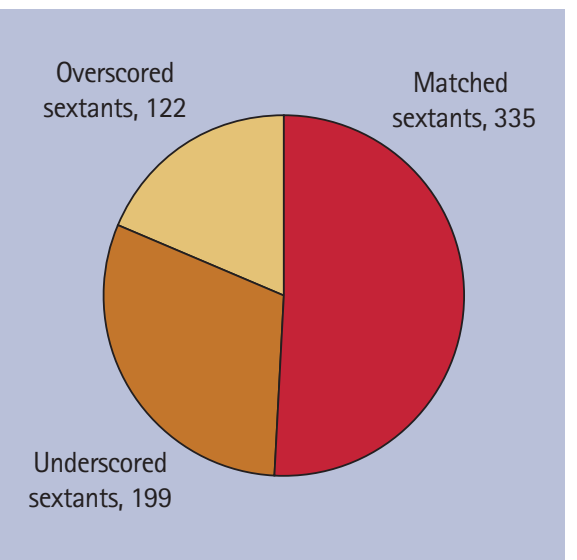

Fig. 2 Comparison of the DwSI and GDP BPE scores

from each general dental practitioner and their disposal. The figures were passed to the consultant on a weekly basis and monthly figures were presented to the director of the service and to the Oral Health Advisory Group (Fig. 1).

\section{Patient feedback}

A patient questionnaire was sent to the first 50 patients referred to the service. The questionnaire was designed to acquire information on how the patient perceived the service and the care that they were receiving. The questionnaire was therefore very subjective but it was the patients' perception of the service that we wished to assess. Patients were given a five-point scale (very poor, poor, don't know, good and very good) with which to answer. The data were anonymous but patients were requested to provide information voluntarily on age, gender and ethnicity. Some questions related to the service information leaflets were also included, along with questions regarding whether the patient minded that they were not seeing a consultant.

\section{GDP feedback}

A questionnaire was also sent to the first 50 referring GDPs. The questionnaire was anonymous but practitioners were invited to provide their practice details. The questionnaire was aimed at assessing some of the concerns raised by the LDC regarding the complexity of the referral form. It also asked for the GDPs' subjective assessment of the care provided. A five-point scale of very poor, poor, don't know, good and very good was used. Questions also assessed the PCT performance and the information 


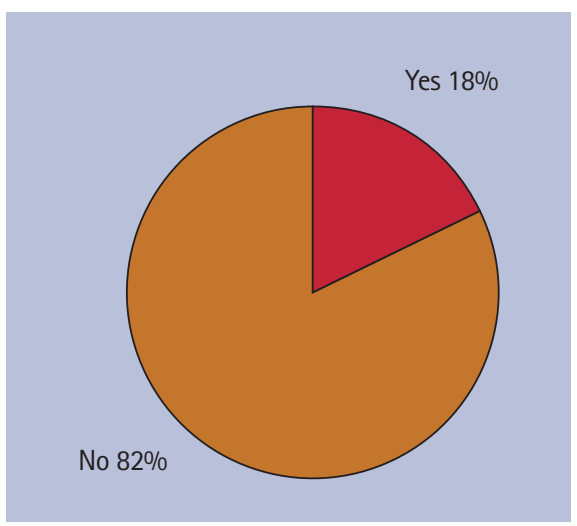

Fig. 3 Does the DwSI service replace the need for a consultant in restorative dentistry? Response from referring GDPs

circulated to GDPs regarding the service framework as well as the periodontal training received by the DwSIs.

A potential concern regarding the provision of this type of service, among the secondary sector, was that the GDPs would see the service as a replacement for the traditional consultant-led service. This question was specifically addressed and 82\% said that it did not replace the consultant service (Fig. 3).

Within a short time of the start of the service, it was notable that some practitioners used the service far more than others (Fig. 4). The data were split off into a subset of practitioners referring more than five patients in order to compare and contrast their attitudes with the larger group that referred fewer than five patients. The data are not presented in this paper.

Additional information on referring practitioners was obtained from the referral management centre.

\section{Clinical data}

The clinical data were captured by Software of Excellence. The service was assessed as a whole and on an individual DwSI basis but only the combined data is presented in this paper.

\section{RESULTS}

\section{Does the service work for the PCT?}

The service was well utilised by the practitioners and referrals were greater than anticipated (Table 1). 428 were referred to the service in the first 12 months. Only 12 patients were passed directly to the consultant. Fifty-five referrals were returned to the GDP due to inadequate information

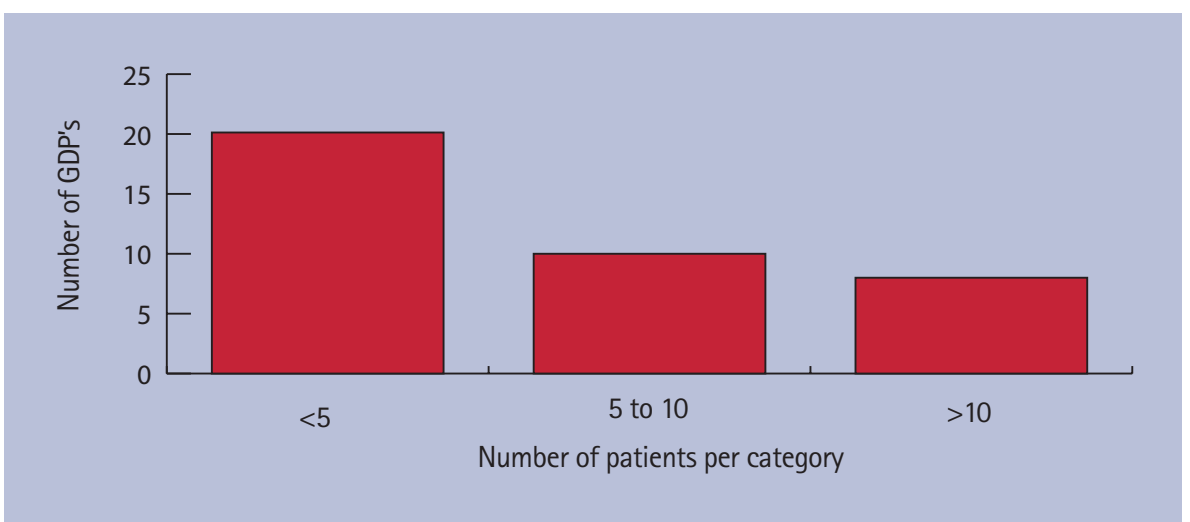

Fig. 4 Number of patients referred by responding GDP

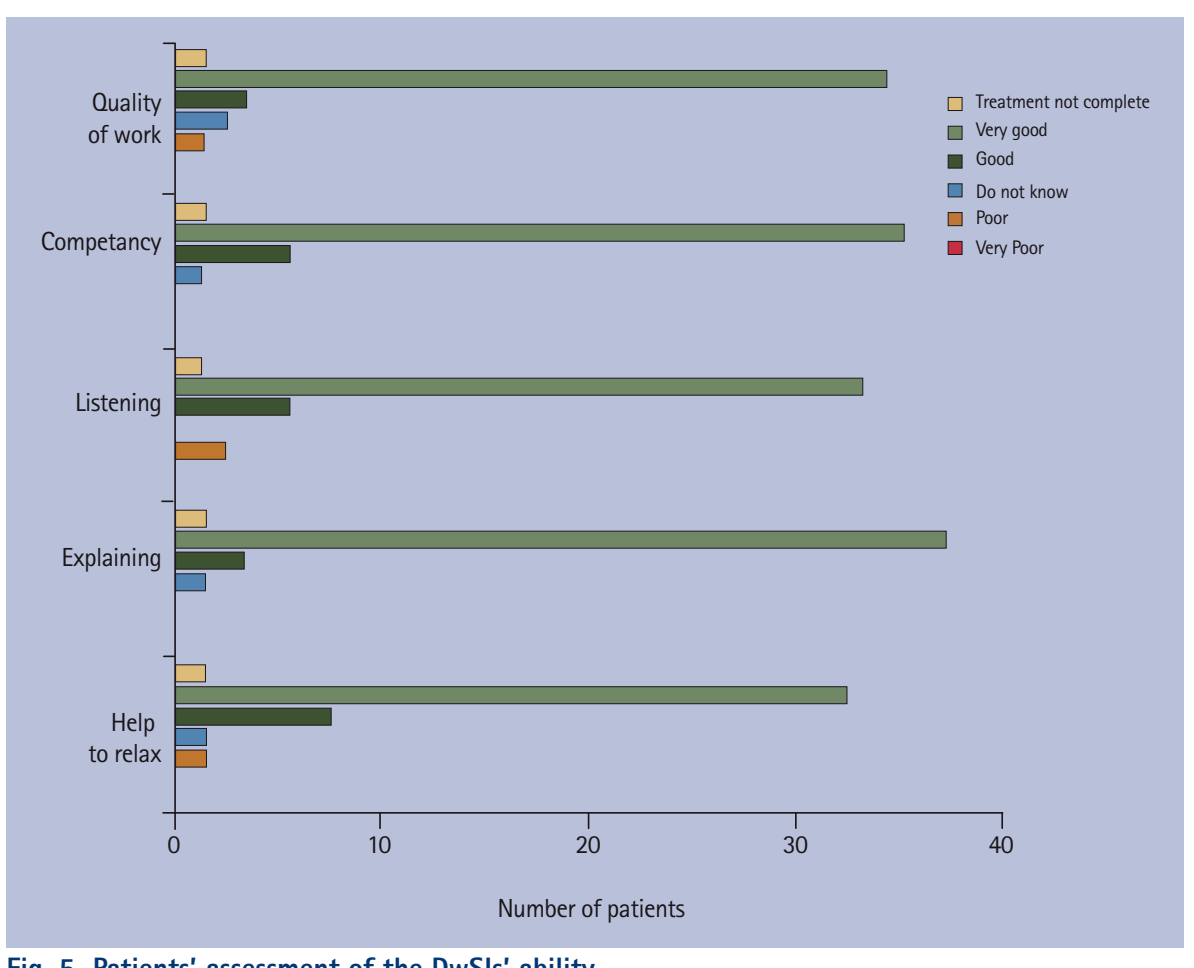

Fig. 5 Patients' assessment of the DwSIs' ability

or due to an inappropriate disease level. Eighteen referrals were rejected as the patient was not a resident of West Sussex. During April and May 2007, 55 patients were not allocated to DwSIs as the PCT closed the service to new referrals pending a countywide oral health needs assessment. A total of 288 patients were therefore seen by the DwSIs. This gave an average of just over five patients seen per session.

$66 \%$ of the patients seen by DwSI were women and this is probably a reflection of the fact that women and men access healthcare in different ways. ${ }^{6}$

$66 \%$ of patients were aged 46-64; $20 \%$ aged $65+; 14 \%$ aged $19-45$. The proportions appear to be in line with the target disease groups.

Analysis of the referral data in collaboration with the DwSI data confirmed that there was an equal distribution of patients across the county.

\section{Does it work for the patients?}

The patient questionnaire had an $84 \%$ return rate. The patients were equally distributed between the five DwSIs.

of the patients returning the questionnaire:

- 95\% said that they understood the role of the DwSI. Only three patients were uncertain whether they had received the DwSI leaflet, the rest clearly recalled receiving it

- 93\% patients were seen within 15 minutes of their appointment time

- $97 \%$ felt that their periodontal problems were addressed by the service

- $83 \%$ rated care as very good. One patient was dissatisfied (Fig. 5) 
- 94\% rated the DwSI communication as good or very good. This validates the emphasis placed on communication during the training programme (Figs. 5 and 6)

- 79\% found it very easy or fairly easy to arrange an appointment with the DwSI

- 30 of the 42 patients did not mind the fact that they were not seeing a consultant. No patients took the opportunity to request to see a consultant.

\section{Does it work for the referring GDPs?}

Thirty-eight practitioners out of 50 returned the questionnaire. This represents a 76\% return rate. Twenty of the practitioners referred fewer than five patients. Eight practitioners had referred more than ten (Fig. 4). Additional data from the RMC revealed that one practice referred 52 patients in the first eight months. The next highest referral practice was from a practice in which one of the DwSIs worked. Forty-three patients were referred from this two-person practice.

Before starting up the service, it was explicit that patients referred from a DwSI practice would not be seen in their own practice. It was agreed that they would be allocated by the RMC to a different DwSI practice. This obviously went against the principle of local access, but it ensured equity for all referring practitioners. It also allowed the DwSI to experience the system from the referring practitioners' aspect.

This approach was fully justified by the fact that one of the DwSI practices referred a high number of patients to the pilot. They would therefore have effectively been in a position to solely look after their own patients and their own practice interests.

Questions 2-6 of the practitioner questionnaire (Figs 7 and 8) relate to the referral form and the ease of completion of the form. The majority of practitioners had no problems completing the form but over 50\% found that it was time-consuming. Thirtyfive out of the 38 practitioners found the referral criteria easy to understand.

In drawing up the referral form, there was some discussion at LDC meetings regarding its content. The LDC felt that the form was too complex. However, as the form was processed by non-dental

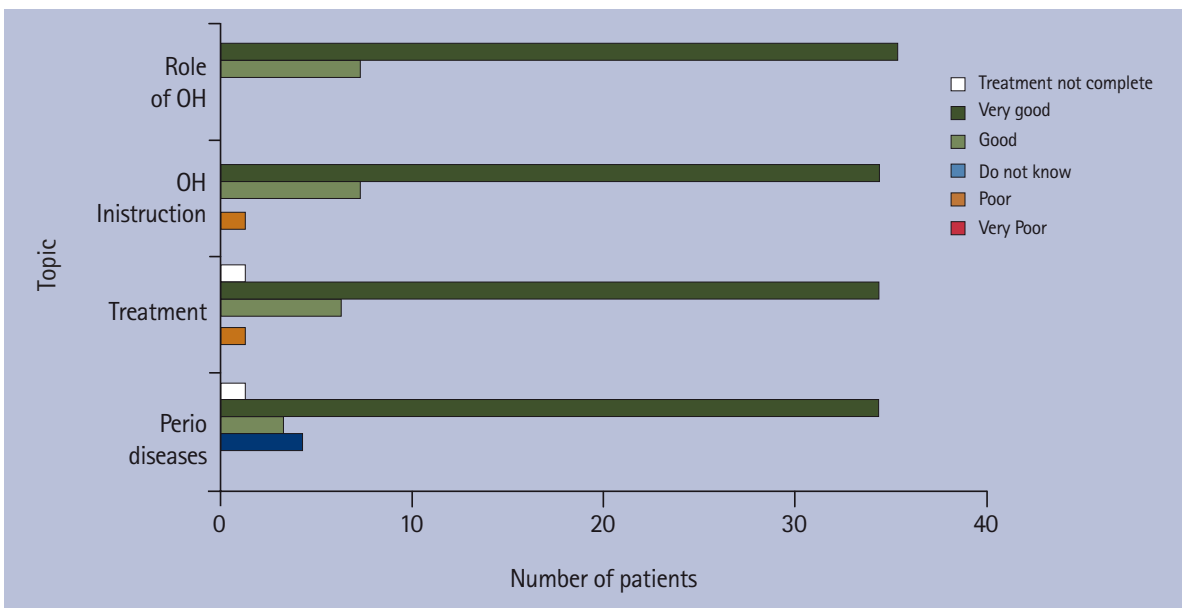

Fig. 6 Patients' perception of the DwSIs' communication

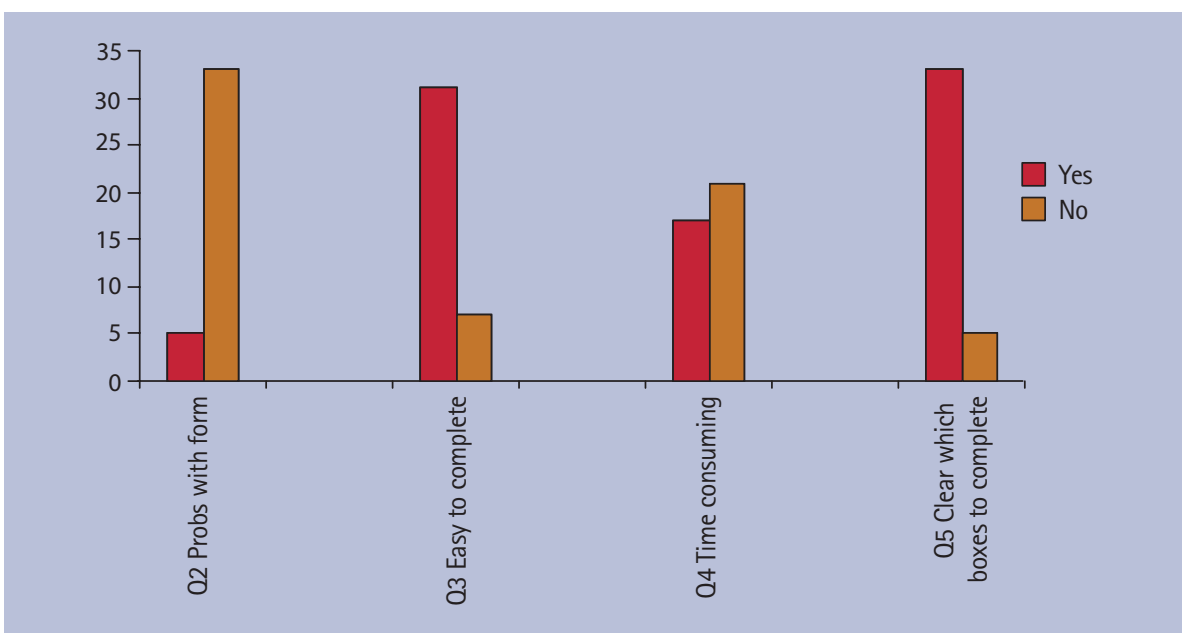

Fig. 7 Questions regarding the referral form

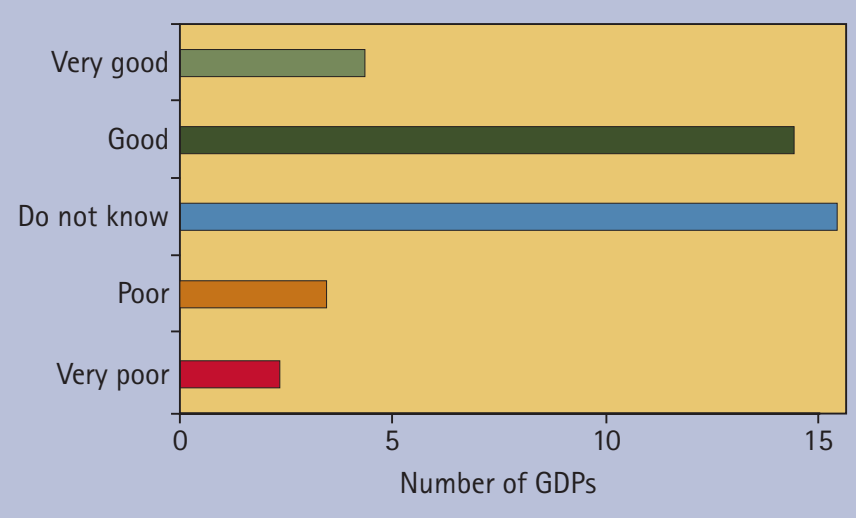

Fig. 8 How the GDPs rate the outcome

clerics in the Referral Management Centre, there was a need to have certain critical information, in order to process the form correctly. The referral form audit indicates that the form was acceptable to the referring practitioners.

Questions 7 and 8 assessed the PCT's ability to fulfil the key performance indicators of the service. Twenty-six practitioners did not receive confirmation of their referral from the PCT within the statutory three weeks. Twenty-five did not receive any confirmation.

Questions 9 and 10 were related to the referral process and appointment allocation system. Twenty-six of the referring practitioners completing the questionnaire (74\%) did not experience any problems with the organisation of an appointment for their patient.

Before starting the service, every practitioner was sent a letter explaining the 
concept of a DwSI, along with information on the referral criteria and pathway for the service. In the GDP audit, two-thirds of practitioners said that they understood the referral pathway.

Several questions addressed the practitioner's assessment of the treatment provided by the DwSI and their perception of the patient's experience of the service. The nature of the questions is very subjective. This is acceptable, as we required their subjective assessment of the service, even if part of their assessment was clinically based. The GDP questionnaires were sent out eight months after the service started, but in hindsight were sent out too soon. Fifteen of the practitioners could not comment on the DwSIs' care as they had not yet seen their patients or because the patients had not completed their periodontal therapy. of those able to comment, the majority were positive about the clinical outcome, their patients' experience and the DwSIs' communication (Figs 9, 10 and 11).

Perhaps the most significant question regarding the GDPs' acceptance of the service was: 'would they continue to use the service?' Thirty-six practitioners (95\%) stated that they would continue to refer to the service. One practitioner did not complete this section and one practitioner said that they would not refer again. The last practitioner did not elaborate on his reasons for this and it was not possible to discern from the questionnaire where the negative experience had arisen.

Practitioners were asked if the service replaced the need for a consultant in restorative dentistry (Fig. 3). Thirty-one practitioners (82\%) said that it did not replace the consultant. The seven practitioners that felt that it did replace the consultant were from the group of GDPs referring more than five patients. The majority of GDPs in this higher-referring subgroup did not train in the UK. It may reflect that these practitioners have a different concept and experience of health care delivery.

When the subset of higher-referring practitioners was analysed separately, $59 \%$ of them still felt that the service did not replace the consultant. This is probably because the role of the consultant in restorative dentistry includes periodontology and incorporates the other restorative disciplines. The ability to undertake a

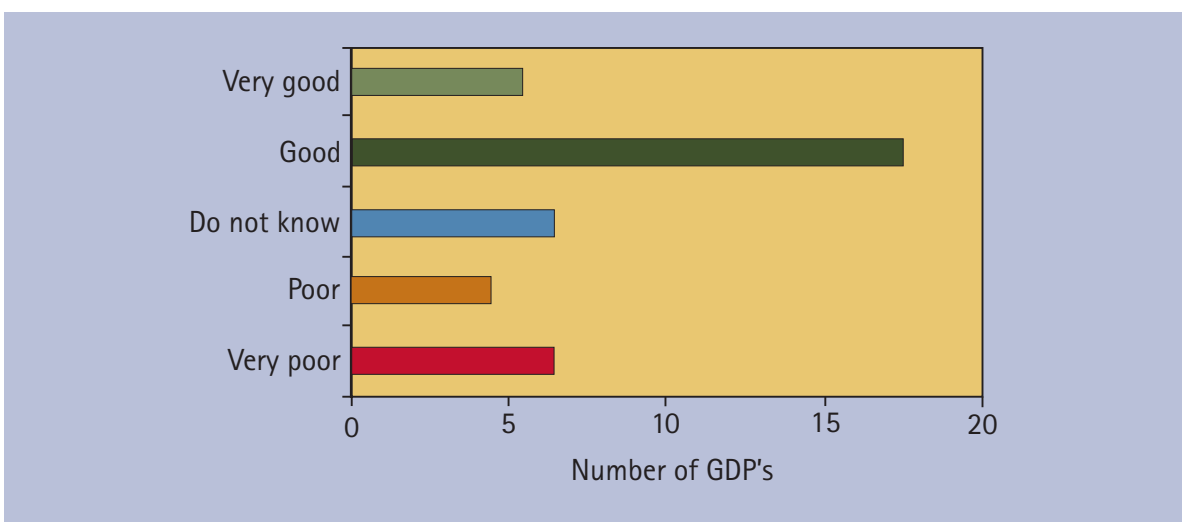

Fig. 9 How the GDPs rate the DwSls' communication

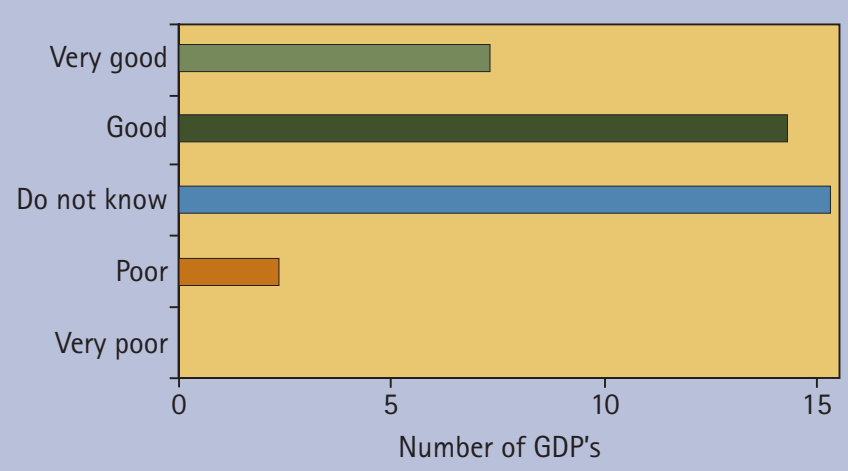

Fig. 10 How the GDPs rate the care provided for the patient

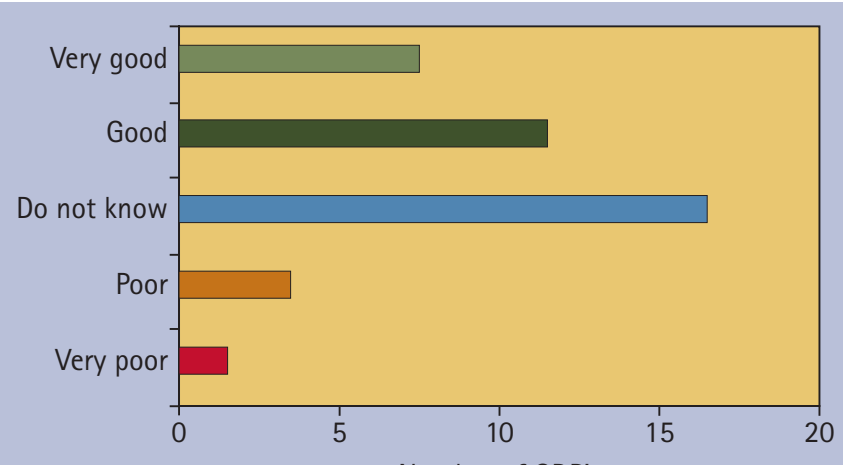

Number of GDP's

Fig. 11 The GDPs' interpretation of the patient experience

whole-mouth assessment is critical when providing treatment planning advice. It is recognised that the consultant's role is diverse and that they are regarded by the patient and GDP as an independent source of information.

The DwSIs were instructed not to give advice regarding treatment planning on non-periodontal issues. The PCT took the view that from a clinical governance and medico-legal perspective, the DwSIs were not in a position to give this advice. The definition of a DwSI includes the need to refer on where necessary, and for this reason there are advantages in the use of a Clinical Network that interacts with the secondary sector. The consultant outreach into the DwSI practice also facilitated dialogue around these areas.

\section{Does it work for the DwSls?}

The DwSI audit revealed the following patient-related data:

- $34 \%$ smokers

- 93\% had plaque scores (PS) in excess of $50 \%$ at referral

- $98 \%$ had bleeding scores in excess of $50 \%$ at referral

- 53\% were diagnosed by the DwSI as having chronic generalised periodontitis

- 30\% had chronic localised periodontitis

- $17 \%$ had aggressive periodontitis. 
The DwSI were instructed to verify the BPE using a WHO or CPITN probe.

When the DwSI BPE assessment data were analysed and compared to the GDP BPE data (Fig. 2), the following became evident:

- There was a complete match between referrer and DwSI in only 18\% of the patients

- Overall 51\% of sextants exhibited a matching BPE

- 19\% of sextants were overscored by the GDP

- $30 \%$ of sextants were underscored.

As there was no calibration exercise carried out between the referring GDPs and the DwSIs, the significance of the information is unknown. There are many factors which influence probing depth measurement. ${ }^{7}$ Its value as a screening tool has been questioned, particularly in the presence of inflammation. ${ }^{7}$ Despite this, periodontal probing is routinely undertaken as part of the process of assessing the patient's disease status. In the context of this audit it was necessary to have baseline data.

The BPE has been recommended for use as a periodontal screening tool in general practice since $1986 .{ }^{8}$ The familiarity and use of the BPE in general dental practice has been questioned in the literature. ${ }^{9}$ Interestingly, the analysis shows that more GDP sextants were underscored for disease than overscored. One might have expected there to be a tendency for referring dentists to overstate rather than understate disease levels, in order for the patient to be accepted into the scheme. It is unlikely that disease would have progressed significantly within the time frame of the referral, as the PCT was required to contact the DwSI practice within three weeks of the receipt of the referral.

In addition, as the DwSI's were trained in periodontal probing, it is likely that they would more accurately reflect the disease level, rather than overscore the disease. In the light of this, the potential underscoring of disease by the practitioner is perhaps of concern.

Regarding the treatment protocol:

- $90 \%$ of the patients were seen within 4-7 visits

- Over $80 \%$ showed a $50 \%$ or higher improvement in their bleeding and plaque scores
- Over 50\% of patients had $80 \%$ of their $5.5 \mathrm{~mm}$ or greater pockets moved into a lower BPE category, making them suitable for maintenance.

Only 11 teeth were extracted during the assessment phase. Six of these were in the smoking group.

$87 \%$ of patients were discharged back to their GDP. The improvement in the plaque and bleeding scores over the treatment time indicated that patients had accepted their role in the management of their own disease. It is hoped that this will be carried on to the maintenance phase.

Patients were considered suitable to return for maintenance if their probing depths were less than $5 \mathrm{~mm}$ or if the probing depth was greater than $5 \mathrm{~mm}$ but did not bleed on probing. Patients with probing depths greater than $5 \mathrm{~mm}$ and with bleeding on probing were referred on to the consultant for further management.

The patients that were referred to the consultant by the DwSI were all from the smoking group.

\section{DISCUSSION}

The DwSI were paid at a rate of $£ 350$ for a 3.5 hour session. This figure was arrived at bearing in mind Guild rate payments and the fee per session paid to medical practitioners with a special interest. As this was a pilot study, funding was kept separate from the dental contract and the scheme did not provide Units of Dental Activity. As part of the service provision, it was agreed that the PCT would fund the attendance and accommodation of the DwSI's at the British Society of Periodontology spring meeting 2007. This was considered to be an essential part of the DwSIs' continuing professional development.

The use of protocols was something that the DwSIs' initially found difficult. Most were self-employed and were used to entire clinical freedom. The concept of being part of a corporate body that dictated their clinical practice was not initially understood. This is now more commonplace in general practices. Once the concept that they were delivering care on behalf of the PCT was understood, the DwSIs appreciated the need for consistency of care so that the service could be audited.

As the service progressed and the effective clinical outcomes became apparent, the DwSI's developed confidence in this patient centred oral hygiene approach. The concept of delaying instrumentation until the plaque scores were low enough was valuable for the DwSI and the patient alike. It highlighted the effect that toothbrushing on its own has on the gingival tissues. The DwSIs began to adapt this approach to their own practices.

The emphasis placed on effective communication appears to have been successful, according to the patients' assessment of the DwSIs' ability to communicate. The time allocation for each appointment clearly facilitated this.

There is obvious scope to refine the protocol, particularly in the light of the evidence of whole mouth versus quadrant root surface debridement. However, there is merit in the quadrant approach, as this allowed for communication and reinforcement of patient-performed oral hygiene over a greater time period.

In the future the role of root surface debridement can be taken up by the hygienist working closely with the DwSI. The DwSI would concentrate on the initial assessment, periodontal treatment planning and re-assessment, thereby increasing efficiency. The re-assessment stage is critical and the DwSIs needed time to acquire the knowledge of assessing the subtle changes seen in resolving gingival tissues. This insight influences the emphasis for the next phase of treatment. The DwSI would need to be able to analyse the hygienist's treatment and be able to discuss the treatment as an equal. This is not possible if the DwSI has not enough hands-on experience of the hygienist role.

There was a clear value in creating a primary-secondary sector clinical network rather than independent sectors. It allowed for effective communication and support for the DwSI during the initial stages. The outreach into GDP5 indicated how important this support was for implementing change. The network was efficient in forwarding appropriate patients to the secondary sector, ensuring that the patient was seen by a clinician with a knowledge and skill level commensurate with their disease status. The outreach reduced referrals to the secondary sector as the DwSI and consultant were able to discuss patient care in the practice. 
It is regrettable that many of the practitioners were unable to answer some of the questions as they had not seen their patients. This obviously dilutes the value of the audit. However, the outcome data indicates the clinical success of the scheme. A followup audit of the patients sent back to their practitioners for maintenance would give a clearer insight into the success of this selfefficacy and behaviour change approach.

\section{CONCLUSIONS}

Audit of this DwSI service indicated that it was highly successful in treating the targeted disease levels and achieving key performance indicators. The patient response seemed to validate the emphasis on oral hygiene and self-efficacy. The treatment protocol appeared to be successful in its outcomes. The subjective feedback from the patient is validated by the outcome data. Once the DwSI has gained enough experience, the service could be made more efficient by undertaking a whole mouth debridement protocol and by extending the service to hygienists within the DwSI practice. There is much merit in developing clinical networks between the primary and secondary sectors in order to liberate the talents of general dental practitioners.

With sincere thanks to: Phil Ower and Graham Smart, specialist periodontists; Stephen Lamber Humble and Graham Gilmour of KSS Deanery; Mike Mulcahy of the Faculty of General Dental Practice (UK). Without their help this project would not have seen the light of day.

This paper is dedicated to the memories of: Sonja Bescoby of Mid Sussex PCT, who always had her vision on the horizon while others were still looking at their own feet. She had the capacity to see the whole picture.

Bernie Kieser, who lectured on the training programme. He has consistently been the strongest advocate of properly performed plaque control. His teaching is the inspiration behind the programme.

Graham Smart, who, with Phil Ower, helped deliver the majority of the periodontal training. His friendship and knowledge is sorely missed.

1. Department of Health. The NHS plan: a plan for investment, a plan for reform. London: Department of Health, 2000.

2. Department of Health. The NHS improvement plan: putting people at the heart of public services. London: Department of Health, 2004

3. Department of Health, Faculty of General Dental Practitioners (UK). Implementing a scheme for dentists with special interests. London: Department of Health, 2004.

4. NHS Primary Care Contracting. Dentists with special interests (DwS/s): a step by step quide to setting up a DwSI service. NHS Primary Care Contracting, 2006.

5. Cheshire PD. Why undertake outreach into general dental practice? Br Dent J 2002; 192: 371-374.

6. Wilkins D Payne S, Granville G, Branney P for Department of Health. The gender and access to health services study: final report. London: Department of Health, 2008.

7. Listgarten M A. Periodontal probing: what does it mean? J Clin Periodontol 1980; 7: 165-176.

8. Mosedale R F, Floyd P F, Smales F C (eds). Periodontology in general dental practice in the United Kingdom. A first policy statement. British Society of Periodontology; 1986.

9. Tugnait A, Clerehugh V, Hirschman P N. Use of the basic periodontal examination and radiographs in the assessment of periodontal diseases in genera dental practice. J Dent 2004; 32: 17-25. 